\title{
Cyclin-dependent kinase 2-associated protein 1 suppresses growth and tumorigenesis of lung cancer
}

\author{
MEI SUN ${ }^{1}$, RUI JIANG ${ }^{5}$, GUAN WANG ${ }^{2}$, CHAO ZHANG $^{3}$, \\ $\mathrm{JINDONG} \mathrm{LI}^{4}, \mathrm{CHENGYAN} \mathrm{JIN}^{4}$ and XINGYI ZHANG ${ }^{4}$ \\ Departments of ${ }^{1}$ Pathology, ${ }^{2}$ Cardiac Medicine, ${ }^{3}$ Ophthalmology and ${ }^{4}$ Thoracic Surgery, \\ The Second Hospital of Jilin University, Changchun 130041; ${ }^{5}$ Department of Orthopedic Surgery, \\ China-Japan Union Hospital of Jilin University, Changchun 130033, P.R. China
}

Received November 18, 2012; Accepted January 11, 2013

DOI: $10.3892 /$ ijo.2013.1813

\begin{abstract}
Cyclin-dependent kinase 2-associated protein 1 (CDK2AP1), a growth suppressor that negatively regulates CDK2 activity, has been implicated in various types of cancer; yet its role in lung cancer remains unclear. In the present study, a lentivirus-based system was used to specifically downregulate or upregulate CDK2AP1 expression. A549 lung cancer cells were treated with RNAi (RNA interference) or lentiviral vectors for overexpression. Ectopic overexpression of CDK2AP1 in A549 cells in vitro greatly impaired their proliferation and colony-forming ability and enhanced their chemosensitivity to cisplatin and paclitaxel and caused cell cycle arrest at G1/S transition accompanied by the reduction of expression of CDK4 and CDK7. Injection of the ectopically CDK2AP1-overexpressing A549 cells into nude mice resulted in growth arrest of solid lung cancer tumors in vivo. Knockdown of CDK2AP1 in A549 cells, however, gave rise to the opposite effects including promoting cell proliferation/ growth, cell cycling in vitro and enhancing tumorigenesis in vivo. These results suggest that CDK2AP1 plays an important role in modulating the growth and tumorigenesis of lung cancer cells and also has significant effects on the chemosensitivity of pulmonary malignancies to chemotherapeutics. Hence, this study extends our knowledge on the relationship between CDK2AP1 and oncogenesis of lung cancer, indicating that CDK2AP1 may serve as a new molecular target for future lung cancer therapy.
\end{abstract}

\section{Introduction}

Better understanding of the molecular mechanisms underlying pulmonary oncogeneisis is critical for the development of optimally therapeutic modalities (1-3). Thus far, numerous

Correspondence to: Dr Xingyi Zhang, Department of Thoracic Surgery, The Second Hospital of Jilin University, 218 Ziqiang Street, Changchun 130041, P.R. China

E-mail: xingyi_zhang@163.com

Key words: lung cancer, cyclin-dependent kinase 2-associated protein 1 , cell cycle, proliferation, chemosensitivity molecules have been indentified to be implicated in pulmonary oncogenesis. Among these molecules, the abnormalities in cell cycle regulatory proteins are common in lung cancer (4). The cell cycle is governed by cyclin-dependent kinases (CDKs) whose activities are regulated positively by cyclins, but negatively by CDK-inhibitors (CKIs) $(5,6)$.

Cyclin-dependent kinase 2-associated protein 1 (CDK2AP1), also named as deleted in oral cancer-1 (DOC-1), is a growth suppressor originally isolated from normal hamster oral keratinocytes by suppression subtractive hybridization (6-8). Human CDK2AP1 is a highly conserved cellular gene and has been mapped to chromosome 12q24 (8). The human CDK2AP1 cDNA is 1.6-kilobase pairs $(\mathrm{kb})$ in length, encoding 115 amino acids with a molecular weight of $12.4 \mathrm{kDa}$ (pI of 9.62) (8). CDK2 activity is thought to play a key role in late $\mathrm{G} 1$ to $\mathrm{S}$ phase progression by phosphorylating and inactivating the retinoblastoma $(\mathrm{Rb})$ protein. Phosphorylated and inactive $\mathrm{Rb}$ allows the transcription of genes under the control of E2F, which are required for DNA replication $(5,6)$. Therefore, the downregulation of CDK2AP1, an inhibitor of CDK 2 and hence G1/S transition, is expected to result in an unregulated cell cycle progression. There is evidence to suggest that CDK2AP1 is implicated in negative regulation of CDK2 activity by sequestering monomeric CDK2 and/or targeting CDK2 for proteolysis (7). CDK2AP1 was shown to interact with DNA polymerase $\alpha /$ primase and/or CDK2 and to mediate phosphorylation of the large p180 subunit, suggesting a regulatory role in DNA replication during $S$ phase of the cell cycle. In addition, recent studies have shown that CDK2AP1 mediates the growth suppressing signal from TGF- $\beta$ (9).

Studies have shown that CDK2AP1 gene is usually expressed in normal human tissues. However, emerging evidence suggests that loss or reduced expression of CDK2AP1 might contribute to the multi-step nature of oral carcinogenesis in many types of malignancies including oral cancer, prostate cancer, esophageal carcinoma, gastric cancer and colorectal cancer (9-15) and that its loss may be an event that is associated with tumor progression. Given the significant association of CDK2AP1 expression with tumorigenesis (7), CDK2AP1 may play a role in oncogenesis and serves as a molecular target for cancer therapy. It is perhaps no surprise that CDK2AP1 also plays a functional role in human 
pulmonary tumorigenesis. However, to date, the specific roles of CDK2AP1 in lung cancers have not yet been reported.

A recently developed technique that was used to specifically down- or upregulate gene expression opens a new avenue for cancer study. The RNA interference (RNAi) technique, a powerful tool for carrying out loss-of-function assay, represents a novel alternative to gene inhibition and provides a new approach for studying cancer gene therapy $(1,2)$. Furthermore, another recently developed technique of ectopic overexpression of a gene adds another indispensible dimension to study of cancer gene therapy. Figueiredo and associates have shown that liposome-based ectopic overexpression of CDK2AP1 in squamous cell carcinoma VII/SF (SCC-VII/SF) significantly induced antitumor responses in an in vivo mouse model of head and neck cancer (16). Ectopic expression of CDK2AP1 in hamster oral carcinoma HCPC-1 cells, which were induced by 7,12-dimethylbenz(a)anthracene, has been associated with growth suppression and a significant antiproliferative effects. Additionally, transfection of CDK2AP1 into HCPC-1 cells significantly increased apoptosis compared with untransfected controls (17).

To study whether and how CDK2AP1 is involved in tumorigenesis of the lung, we adopted the lentiviral vector-mediated RNAi and upregulation system to achieve highly stable silence and ectopic overexpression of CDK2AP1 in A549 cells. The impacts of CDK2AP1 suppression or overexpression on proliferation, cell cycling and chemosensitivity of A549 cells were investigated.

\section{Materials and methods}

Animals. Male Balb/c nude mice (Shanghai SLAC Laboratory Animal Co. Ltd., Shanghai, China) between 4- and 6-week of age were used for the study. These mice were kept under specific pathogenic free (SPF) conditions and 12-h light-dark cycles, with free access to water and standard laboratory diet. Animal manipulation was approved by the Animal Care and Use Ethics Committee of the Second Hospital of Jilin University, China.

Lentiviral vector construction and transfection. For CDK2AP1 expression vector construction, the fragment of CDK2AP1 CDS was amplified and inserted into MCS of pCDH cDNA cloning vector. Blank $\mathrm{pCDH}$ vector was used as a control. For CDK2AP1 RNAi, shRNA cassette against human CDK2AP1 gene was designed based on the CDK2AP1-specific targeting sequence 5'-CATGGCAACGTCTTCACAGTA-3'; Scrambled sequence 5'-AATGTACTGCGCGTGGAGA-3' was used as negative control. The shRNA sequences were synthesized, annealed and ligated into pLKO vector.

For lentivirus packing, the re-constructed pCDH-CDK2AP1, pCDH-Control, pLKO-siCDK2AP1 and pLKO-siControl were co-transfected into $293 \mathrm{~T}$ cells together with packing helper plasmids. The lentiviral particles were then harvested from the culture medium $96 \mathrm{~h}$ post-transfection and then subjected to ultracentrifugation. The constructed lentivirus was referred as Lv-CDK2AP1 for overexpression of CDK2AP1, or Lv-siCDK2AP1 for specific interfering of CDK2AP1.

Human lung adenocarcinoma cell line A549 (Cell Bank of Chinese Academy of Sciences, Shanghai, China) was maintained in Dulbecco's modified Eagle's medium (DMEM) supplemented with $10 \%$ fetal bovine serum $(\mathrm{FBS})$ at $37^{\circ} \mathrm{C}$ in a humidified atmosphere of $5 \% \mathrm{CO}_{2}$. For cell infection, $40 \%$ confluent A549 cells were incubated with si-CDK2AP1, si-control, CDK2AP1 and control lentivirus for $96 \mathrm{~h}$, respectively, with a replacement of medium $24 \mathrm{~h}$ after treatment. Lentivirus transduction efficiency was determined by fluorescence microscopy.

Western blot analysis. Cells were harvested $72 \mathrm{~h}$ after lentivirus transduction and Western blot analysis was subsequently performed. Cells were lysed by pre-cooled lysis buffer $(10 \mathrm{mM}$ Tris-HCl, pH 7.4, 1 mM EDTA, 0.1\% Triton X-100, 0.1\% SDS). The protein concentration was analyzed by Bradford assay kit (Pierce, Rockford, IL, USA). Thirty microgram protein extracted from cells was loaded on $10 \%$ polyacrylamide gel and electrophorised at $30 \mathrm{~mA}$ for $2 \mathrm{~h}$. The resulting membrane was blocked in 5\% non-fat dry milk blocking buffer and then probed with polyclonal antibodies against the CDK2AP1 (Santa Cruz Biotechnology, Santa Cruz, CA), GAPDH (Sigma Chemical Co., St. Louis, MO, no. G8795), CDK4 (Cell Signaling Technology, no. 2906), CDK7 (Cell Signaling Technology, no. 2090) and anti- $\mathrm{Rb}$ (phospho S780) antibody (Abcam, ab47763) respectively overnight at $4^{\circ} \mathrm{C}$. The membrane was washed three times with Tris-buffered saline Tween-20 (TBST), followed by incubation for $2 \mathrm{~h}$ with anti-mouse IgG at a 1:5000 dilution (Santa Cruz). The membrane was developed using enhanced chemiluminescence (Amersham Life Science, Arlington Hts, IL).

Real-time PCR analysis. Total RNAs were extracted using TRIzol reagent (Invitrogen, Carlsbad, CA). The reverse transcription reactions were carried out following the protocol of the M-MLV Reverse Transcriptase (Promega Corp., Madison, WI). The primers used were as follows: for CDK2AP1, 5'-AAG AGCAACCCACCAAACC-3' and 5'-ATCAACTTACAATAA ACGCAGAAC-3'; for actin, 5'-GGCGGCACCACCATGTA CCCT-3' and 5'-AGGGGCCGGACTCGTCATACT-3'. The relative mRNA expression of CDK2AP1 was calculated by the $2^{-\Delta \Delta C t}$ method, using actin mRNA level for normalization. All samples were analyzed in triplicates.

Cell proliferation assay by methylthiazoletetrazolium. Cells were trypsinized $96 \mathrm{~h}$ post-lentivirus treatment, resuspended, seeded into 96 -well plate and incubated at $37^{\circ} \mathrm{C}$. The number of viable cells in all groups was counted at indicated times. At each time point, $10 \mu 1$ 3-(4,5-dimethylthiazol-2-yl)-2, 5-diphenyltetrazolium bromide (MTT) (Dingguo Biotechnology) at $5 \mathrm{mg} / \mathrm{ml}$ was added into each well. The plate was incubated for $4 \mathrm{~h}$, washed and added with $100 \mu \mathrm{l}$ of dimethyl sulfoxide. The absorbance was measured at $490 \mathrm{~nm}$. Each experiment was repeated three times.

BrdU cell proliferation assay. The BrdU Cell Proliferation Assay kit was obtained from Chemicon International (Temecula, CA). Cells were re-plated in a 96-well plate at $2 \times 10^{4}$ cell $/ \mathrm{ml}$ $96 \mathrm{~h}$ after lentivirus treatment. BrdU was added in each well of the plates and incubated for $2 \mathrm{~h}$, followed by fixation and wash. The anti-BrdU monoclonal and the goat anti-mouse IgG, peroxidase conjugate antibodies were used successively. The plate was added with TMB peroxidase substrate for reaction and read at dual wavelength of $490 \mathrm{~nm}$. 

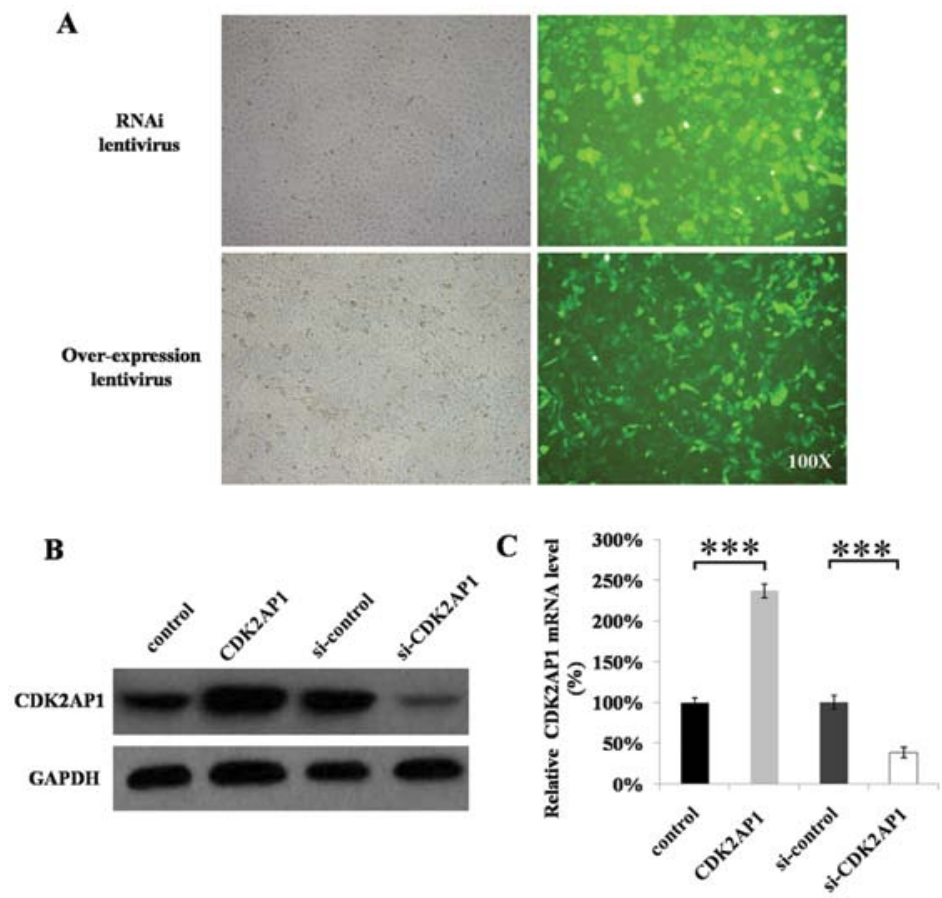

Figure 1. Analysis of lentivirus-mediated silencing or overexpression of CDK2AP by real time RT-PCR and western blotting. (A) Four different lentiviral constructs containing si-CDK2AP1, si-control, CDK2AP1 or control sequences, respectively, were transduced into human lung cancer A549 cells. To determine the transfection efficiency of A549 cells with lentiviruses, fluorescence microscopy was performed $72 \mathrm{~h}$ after infection. Fluorescence photomicrographs of GFP-positive A549 cells infected by CDK2AP1 RNAi (upper) or overexpression (lower) lentivirus are shown. (B) Total mRNA was extracted and analyzed by real-time PCR. The level of CDK2AP1 mRNA expression was normalized with that of $\beta$-actin mRNA expression. (C) Cell lysates were subjected to SDSPAGE and immunoblotted with anti-CDK2AP1 and anti-GAPDH antibody. GAPDH was simultaneously immunodetected to verify the loading of similar amounts of cell lysates. ${ }^{* * *} \mathrm{P}<0.001$ in comparison with control or si-control.

Colony formation assay. After infection, cells in all four groups were seeded into a 6-well plate at a concentration of 200 cells per well and maintained at $37^{\circ} \mathrm{C}$ for 14 days. The culture medium was changed every 2-3 days. At the end of incubation, the plate was washed with PBS, fixed with paraformaldehyde, stained with Giemsa stain (Sigma) for $10 \mathrm{~min}$ and washed with $\mathrm{ddH}_{2} \mathrm{O}$, sequentially. The stained cells were photographed with a digital camera. The number of colonies in each well was counted and analyzed.

Flow cytometric analysis. Cells in all four groups were harvested by centrifugation at 1,200 rpm for $5 \mathrm{~min} 48 \mathrm{~h}$ after infection. The cell pellets were washed with cold PBS, fixed with $70 \%$ ethanol and harvested after centrifugation. Then the pellets were resuspended with PBS and filtrated through 400-mesh membrane. The cell samples were stained with propidium iodide (PI) (Sigma)/RNase/PBS (100 $\mu \mathrm{g} / \mathrm{ml}$ PI and $10 \mu \mathrm{g} / \mathrm{ml} \mathrm{RNase}$ A) solution at $4^{\circ} \mathrm{C}$ for $30 \mathrm{~min}$ in dark. Stained samples were analyzed by a FACsCalibur II sorter and CellQuest FACS system (BD Biosciences, San Diego, CA, USA). The percentage of cells in each cell cycle phase was determined. All samples were measured in triplicates.

Assessment of the effects on the chemosensitivity to cisplatin and paclitaxel. A549 cells were seeded in a 6-well plate at a concentration of $5 \times 10^{4}$ cells/well for $24 \mathrm{~h}$ and then infected with CDK2AP1 overexpression lentivirus. After 96-h incubation, cells were harvested and re-seeded in a 96-well plate and cultured for $24 \mathrm{~h}$. For chemosensitivity assessment, parental cells, control or CDK2AP1-overexpressing lentivirus infected cells were treated with cisplatin $(10 \mu \mathrm{g} / \mathrm{ml})$ or paclitaxel $(100 \mu \mathrm{mol} / \mathrm{ml})$ for $24 \mathrm{~h}$, respectively and subjected to cell viability analysis by MTT assay.

Tumorigenesis. Tumor cell inoculation into the nude mice was performed using the modified technique previously described by us $(1,2)$. A549 cells $\left(5 \times 10^{6}\right)$ infected with lentiviruses containing si-CDK2AP1, si-control, CDK2AP1 and control sequences were injected subcutaneously into the back of male nude mice ( $\mathrm{n}=8$ per group). The development and growth of solid tumors were monitored by measuring tumor size at 0,10 , 17 and 24 days after inoculation. Tumor volume was calculated by the formula: $\mathrm{V}=0.5 \times \mathrm{L} \mathrm{x} \mathrm{W} 2\left(\mathrm{~mm}^{3}\right)$, where $\mathrm{V}, \mathrm{L}$ and $\mathrm{W}$ represent the volume, length and width of tumors, respectively. The tumor volume in each group was analyzed at $0,10,17$ and 24 days after inoculation. On day 24, mice were euthanized and tumors were excised for measurement of tumor weight.

Statistical analysis. Data are expressed as the mean \pm SD. Student's t-test was performed to evaluate inter-group differences. $\mathrm{P}<0.05$ was taken as statistical significance threshold. All statistical analyses were performed using Prism 5 (GraphPad Software Inc.).

\section{Results}

Efficacy of lentivirus-mediated RNAi and ectopic overexpression of $C D K 2 A P 1$. Fluorescence microscopy examination showed that $>90 \%$ cells were successfully infected as visualized by the GFP tag in lentiviruses (Fig. 1A). The overexpression 
A

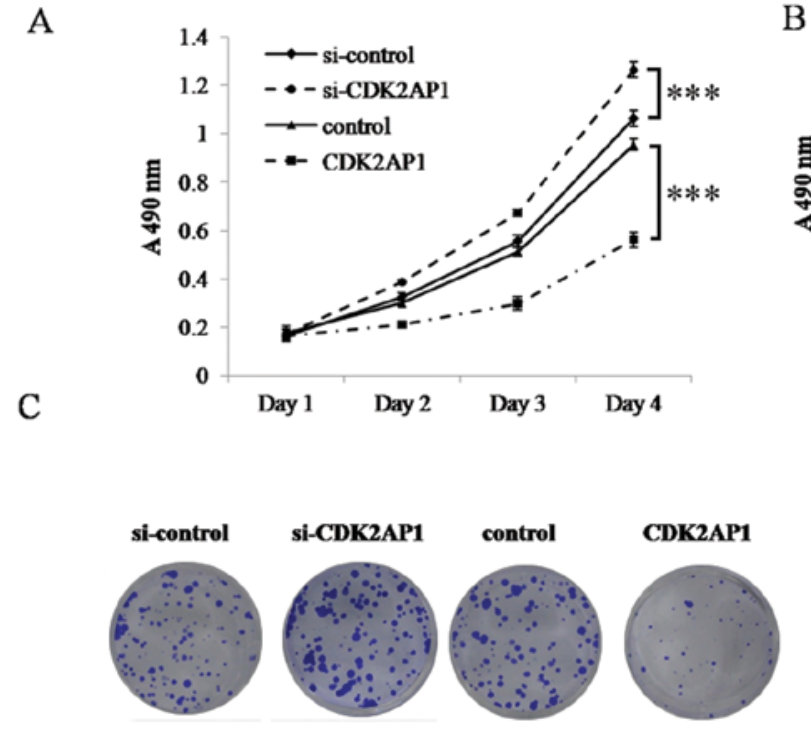

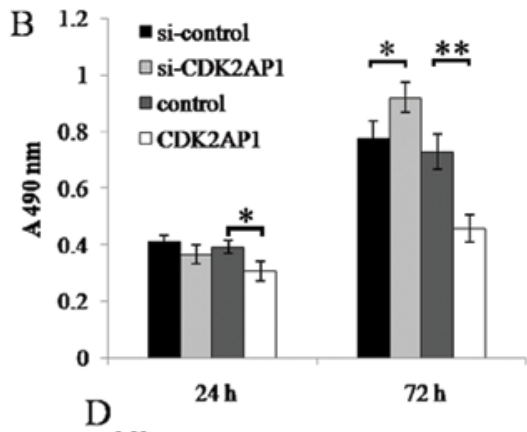

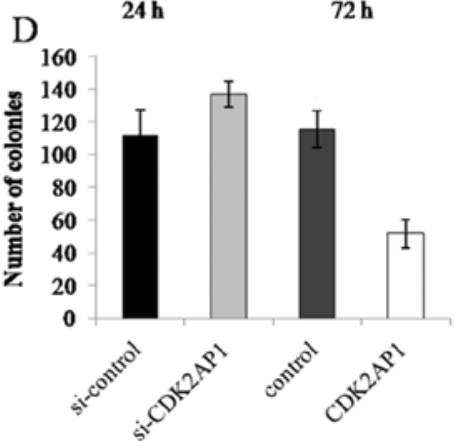

Figure 2. Effects of CDK2AP1 expression on A549 cell growth in vitro. A549 cells were treated with four different lentiviruses for 96 h and re-seeded in 96-well plate for MTT (A) and BrdU (B) assays. Cell proliferation is expressed as an absorption value of optical density at 490 nm, which is fitted on the $\mathrm{y}$-axis, the time of cell growth as labeled on the x-axis. (C) Colony formation of A549 cells. Lv-si-CDK2AP1, Lv-si-control, Lv-CDK2AP1 and Lv-control transduced A549 cells in 6-cm flat plates were cultured for 14 days and stained with Giemsa. (D) Quantitative analysis of colony formation. ${ }^{*} \mathrm{P}<0.05,{ }^{* *} \mathrm{P}<0.01$ and ${ }^{* * *} \mathrm{P}<0.001$ in comparison with control or si-control.

or shRNA-containing constructs have successfully down- or upregulated the expression levels of CDK2AP1 gene (Fig. 1B and C). The levels of CDK2AP1 mRNA transcripts in mock control did not significantly differ from si-control. The levels of CDK2AP1 mRNA transcripts in A549 cells transduced with si-CDK2AP1 lentivirus were significantly lower than that transduced with si-control (Fig. $1 \mathrm{~B}, \mathrm{P}<0.01$ ), whereas the levels in A549 cells transduced with overexpression lentiviral construct were significantly higher than that transduced with mock control (Fig. 1B, $\mathrm{P}<0.01$ ). Western blotting showed that the expression levels of CDK2AP1 protein in A549 cells transduced with si-CDK2AP1 lentivirus were significantly lower, but significantly higher in that ransduced with overexpression lentiviral construct than those in the controls (Fig. 1C, $\mathrm{P}<0.01$ ), suggesting that the si-CDK2AP1 lentiviral contruct can effectively downregulate CDK2AP1 gene expression at both mRNA and protein levels in A549 cells, whereas the CDK2AP1 lentiviral construct can effectively upregulate CDK2AP1 gene expression at both mRNA and protein levels in A549 cells.

Impact of down-or upregulation of CDK2AP1 on cell growth in vitro. To explore the role of CDK2AP1 in the growth of A549 cells, the growth dynamics of A549 cells transduced with si-CDK2AP1 and CDK2AP1 lentiviral construct was determined by MTT and BrdU cell proliferation assay. MTT assay showed that, during the 4-day incubation period, the growth of control A549 cells did not significantly differ from si-control A549 cells and both had strong proliferation activity. However, the growth of CDK2AP1 knockdown cells was significantly faster than that of si-control cells, whereas that of CDK2AP1 overexpression cells was significantly slower than that of mock control at day 4 ( $\mathrm{P}<0.001$, Fig. $2 \mathrm{~A})$. BrdU incorpo- ration assay revealed that after $72 \mathrm{~h}$ of incubation, knockdown of CDK2AP1 resulted in increased DNA synthesis $(\mathrm{P}<0.05)$, as compared with si-control. In contrast, CDK2AP1 overexpression significantly inhibited DNA synthesis $(\mathrm{P}<0.001)$.

To evaluate the relative long-term effect of CDK2AP1 knockdown or overexpression, the colony formation ability of A549 cells transduced with different lentivirus was studied as well. Quantitative analysis of colony counts showed that following incubation for 14 days, the colony number of control A549 cells was indistinguishable from that of si-control A549 cells. The colony number of Lv-si-CDK2AP1 infected A549 cells was significantly higher than that of control, whereas the colony count of Lv-CDK2AP1 infected A549 cells was significantly slower than that of si-control (Fig. 2C and D). Therefore, these results suggest that downregulation of CDK2AP1 gene expression enhances the growth of A549 cells, whereas ectopic overexpression of CDK2AP1 gene inhibits A549 cell growth in vitro.

Effects of down- or upregulation of CDK2AP1 on cell cycle distribution. To explore the potential mechanism underlying the action of CDK2AP1 on the growth of A549 cells, the cell cycling patterns of CDK2AP1 knockdown and overexpression of A549 cells were determined by flow cytometric analysis. As shown in Fig. 3, the CDK2AP1 RNAi construct resulted in a substantial decrease in G1-phase and an increase in G2/M phase populations $(\mathrm{P}<0.001)$. In contrast, CDK2AP1 overexpression construct caused an elevated G1-phase proportion and a reduced $\mathrm{G} 2 / \mathrm{M}$ phase proportion $(\mathrm{P}<0.01)$. Moreover, there was an obvious impairment in the frequency at $\mathrm{S}$ phase of Lv-CDA2AP1 transduced cells in comparison to mock control. Overall, these results suggest that CDK2AP1 plays an important role in cell cycle control for A549 cells. 


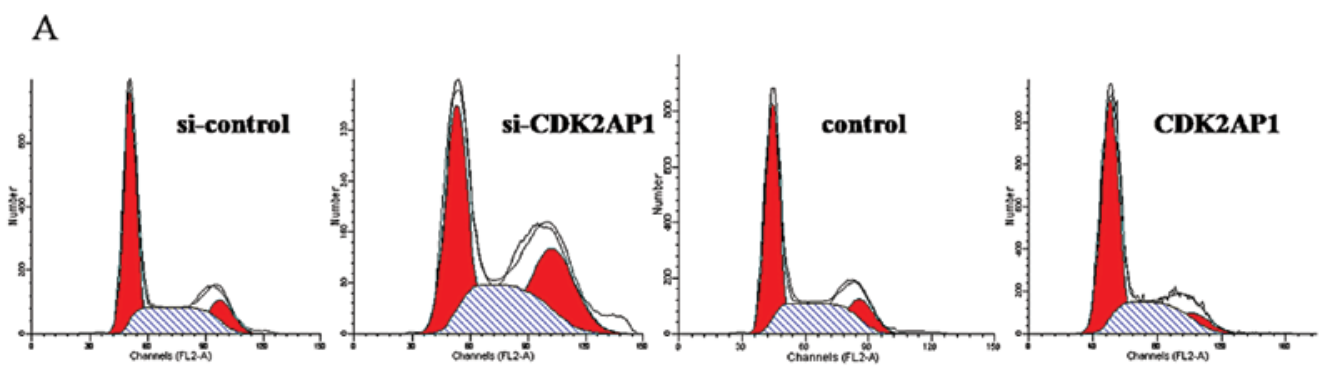

B

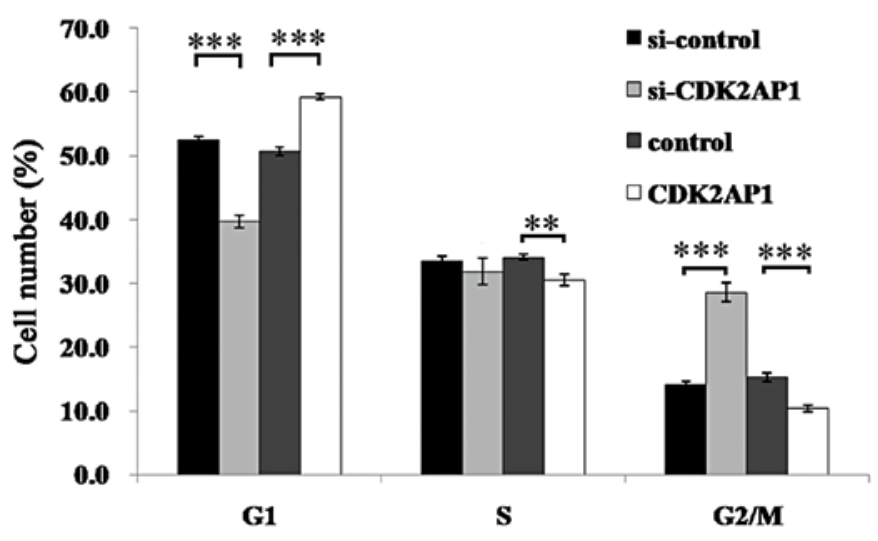

Figure 3. Flow cytometry analysis of A549 cell cycling. The Lv-si-CDK2AP1, Lv-si-control, Lv-CDK2AP1 and Lv-control transduced A549 cells were incubated for $96 \mathrm{~h}$ followed by fixation with $70 \%$ ethanol and PI staining. (A) Flow cytometry histograms of A549 cells following lentivirus transduction in four groups. (B) Analysis of the subpopulations of cells in cell cycle phases $\mathrm{G} 1, \mathrm{~S}$ and $\mathrm{G} 2 / \mathrm{M} .{ }^{* * *} \mathrm{P}<0.01$ and ${ }^{* * * *} \mathrm{P}<0.001$ in comparison with control or si-control.

A

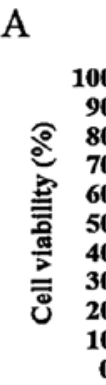

CDK2AP1

Paclitaxel

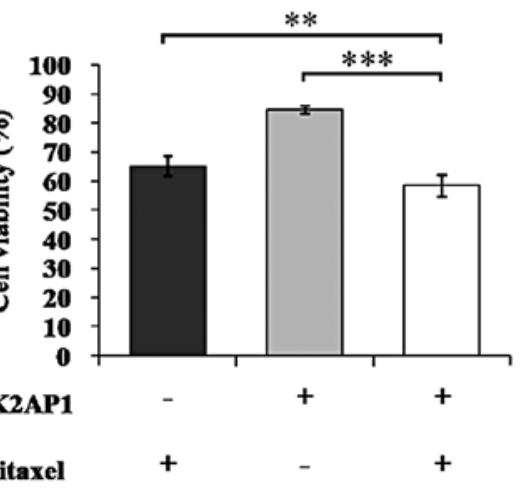

B

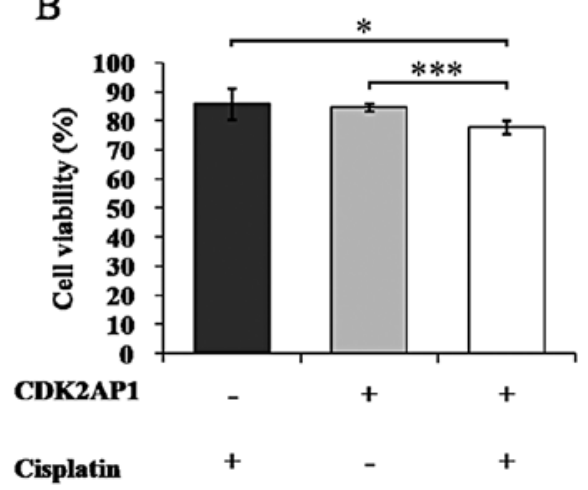

Figure 4. Effects of CDK2AP1 overexpression on chemosensitivity of A549 cells. After 96-h infection with CDK2AP1 overexpressing lentivirus, A549 cells were re-seeded in a 96 -well plate, followed by cisplatin $(10 \mu \mathrm{g} / \mathrm{ml})$ and paclitaxel $(100 \mu \mathrm{mol} / \mathrm{ml})$ treatment for $48 \mathrm{~h}$. (A) Cell viability detection in three different treatments: paclitaxel, CDK2AP1 overexpression lentivirus and the combination of both. (B) Cell viability detection in three different treatments: cisplatin, CDK2AP1 overexpression lentivirus and their combination. ${ }^{*} \mathrm{P}<0.05,{ }^{* *} \mathrm{P}<0.01$ and ${ }^{* * * *} \mathrm{P}<0.001$.

Effects of CDK2AP1 overexpression in A549 cells on chemosensitivity. Cisplatin and paclitaxel are commonly used in lung cancer treatment and thus improvement of their chemosensitivity is critical (18). Here we combined the treatment of CDK2AP1 overexpression lentivirus with cisplatin or paclitaxel in A549 cells to evaluate its effects on chemosensitivity (Fig. 4). Notably, the viability of A549 cells treated with the combination of Lv-CDK2AP1 and paclitaxel was significantly decreased than that with Lv-CDK2AP1 $(\mathrm{P}<0.001)$ or paclitaxel alone $(\mathrm{P}<0.01)$, which was $58.5,84.6$ and $65.2 \%$, respectively $(\mathrm{P}<0.01$, Fig. 4A). Similar results were observed in the combination treatment of Lv-CDK2AP1 and cisplatin (Fig. 4B).
Therefore, CDK2AP1 overexpression can drastically enhance the chemosensitivity of A549 cells to paclitaxel and cisplatin.

Effects of down- or upregulation of CDK2AP1 on tumorigenesis. To determine the role of CDK2AP1 in tumorigenicity, CDK2AP1 knockdown or overexpressing A549 cells were injected subcutaneously into athymic nude mice (Fig. 5). The tumor growth curves and tumor weight results showed that the developed solid tumor in CDK2AP1 overexpression group grew significantly slower than the controls after 24 days of inoculation, whereas the tumors in CDK2AP1 knockdown group grew significantly faster than the controls, being well 

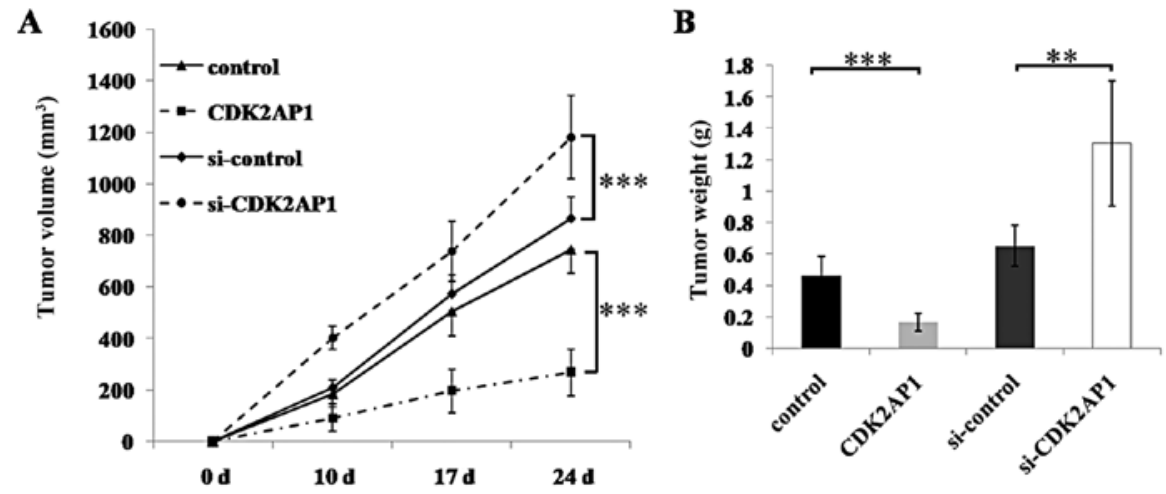

Figure 5. Effects of downregulation or epitopic overexpression of CDK2AP1 gene on the development and growth of inoculated A549 lung cancer cells in vivo. Mice were inoculated with Lv-si-CDK2AP1, Lv-si-control, Lv-CDK2AP1 and Lv-control transduced A549 cells and euthanized 24 days post-inoculation. (A) Tumor growth curve of A549 lung tumors. (B) Quantitative measurement of tumor weights. ${ }^{* *} \mathrm{P}<0.01$ and ${ }^{* * * *} \mathrm{P}<0.001$ in comparison with control or si-control.

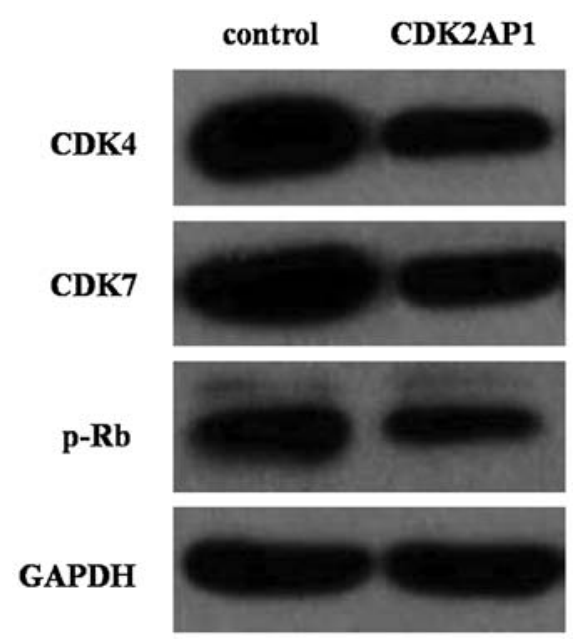

Figure 6. Effects of CDK2AP1 overexpression on cell cycle regulators. The protein levels of CDK4, CDK7 and $\mathrm{p}-\mathrm{Rb}$ were examined via western blot assays. GAPDH was used as loading control.

in agreement with the results of cell proliferation and colony formation in vitro. These results demonstrate that downregulation of CDK2AP1 expression enhanced tumorigenicity of A549 cells, whereas ectopic overexpression of CDK2AP1 gene decreased tumorigenicity of A549 cells in vivo.

Effects of down- or upregulation of CDK2API on cell cycle regulators. CDK2AP1 is known to be associated with CDK2 activity during G1-S phase. To explore the regulator mechanism of CDK2AP1 on A549 cell cycle progression, western blotting was performed on Lv-CDK2AP1 transduced cells. Fig. 6 showed that overexpression of CDK2AP1 resulted in significant decrease in the expression of CDK4, CDK7 as well as the retinoblastoma tumor suppressor gene product $(\mathrm{p}-\mathrm{Rb})$ that plays a pivotal role in G1- to S-phase cell.

\section{Discussion}

In the present study, we determined the role of CDK2AP1 in the growth of A549 cells, a cell line that was initially derived from a highly malignant human lung adenocarcinoma. Using the constructed lentiviral vectors containing CDK2AP1
shRNA or full-length CDK2AP1 cDNA, we infected A549 cells to silence endogenous CDK2AP1 or overexpress CDK2AP1 and investigated systemically the impacts of CDK2AP1 down- or upregulation on lung cancer growth and chemosensitivity in vitro and in vivo. Our results revealed that ectopic overexpression of CDK2AP1 in A549 cells can greatly impair the proliferation and colony-forming ability and enhance the chemosensitivity to cisplatin and paclitaxel in vitro. Notably, we found that CDK2AP1 overexpression caused cell cycle arrest at G1/S transition of A549 cells, as evidenced by the accumulation of G1 phase. Injection of the ectopic overexpressing A549 cells into nude mice resulted in inhibited growth of solid lung tumors in vivo. Consistently with the aforementioned results, knockdown of CDK2AP1 in A549 cells gave rise to the opposite effect on proliferation/ growth, cell cycling and tumorigenesis in vivo. Collectively, these findings not only lend weight to the growing body of evidence that CDK2AP1 is involved in cancer development and progression, but represent the first report that CDK2AP1 is a novel proliferation regulator in lung cancer.

Our data are consistent with previous studies, which have demonstrated the antitumor activity of CDK2AP1 in various malignancies $(11,15,16,19-21)$. In addition, accumulated evidence has indicated that the expression levels of CDK2AP1 may be used as an indicator in diagnostics for the invasiveness and prognosis of oral squamous cell carcinoma, esophageal squamous cell carcinomas and gastric cancer tissues in humans $(10,14)$. Moreover, a recent study revealed that targeted disruption of murine Cdk2ap1 resulted in embryonic lethality with a high penetration rate, which highlights the essential role of CDK2AP1 in the mammal (22).

Cancer research has recently devoted the collective efforts toward an understanding of the molecular regulation and functional significance of cell cycle regulators in the pathogenesis and development of cancers $(4,11,13)$. It has been demonstrated that CDK2 is an important candidate target for therapeutic intervention $(23,24)$. In this study, attention is also paid to the issues regarding association between the antitumor activity of CDK2AP1 and expression of other cell cycle regulators and our finding shows that ectopic overexpression of CDK2AP1 reduces the expression of CDK4, $\mathrm{CDK} 7$ and $\mathrm{p}-\mathrm{Rb}$ in A549 cells. Therefore, we would like to put 
forward a tentative hypothesis that the antitumor activity of CDK2AP1 in lung cancer may act via a mechanism impacting on other cell cycle regulators in addition to CDK2.

In this study, to evaluate the potential value of CDK2AP1 in lung cancer treatment, we examined the chemosensitivity of A549 cells to cisplatin and paclitaxel, which are commonly used in clinical study (18). Many studies have focused on increasing the chemosensitivity of lung cancer cells to chemotherapeutics. Kim and associates have shown that p12CDK2 mediates DNA damage responses induced by cisplatin in mouse oral/head and neck cancer model (25). Our finding represents the first report that CDK2AP1 overexpression can enhance the chemosensitivity to cisplatin and paclitaxel, suggesting that CDK2AP1 may be a potential molecular target in lung cancer therapy.

Several growth factors or growth suppressors have been implicated in cell proliferation and growth as well as invasionmetastasis cascade of malignant tumors $(1,2,13)$. CDK2AP1 has been selected as a functional target of gene therapy for human colorectal cancer and mouse model of head and neck cancer $(12,15,16)$. Our results show that modulation of CDK2AP1 gene expression in A549 cells can affect cell growth and chemosensitivity of lung cancer in vitro and/or in vivo, which is consistent with previous reports $(16,21)$.

Emerging evidence suggests that several factors are implicated in genesis of lung cancer, such as new fusion genes, changing expression level of p53, growth factors, MED19 (a subunit of mediator complex), cytokines and chemokine receptors and STAT3 (signal transducer and activator of transcription 3) $(1,2)$. It has been suggested that CDK2AP1 suppresses malignant biological interactions between prostate cancer and bone cells and modifies the androgen-responsive pathway function $(11,13)$. However, to date, the issue as to whether and how CDK2AP1 interplays with other regulators is poorly understood and further investigation is warranted to elucidate the mechanism underlying the action of CDK2AP1.

In conclusion, our findings strongly suggest that CDK2AP1 facilitates important regulatory roles in lung cancer cell proliferation and cell cycle progression of lung cancer and also has significant effects on the chemosensitivity of pulmonary malignancies. Hence, this study extends our current knowledge on oncogenesis of lung cancer and indicates that CDK2AP1 may serve as a new molecular target for lung cancer therapy.

\section{Acknowledgements}

This study was supported by the grant from the Natural Science Foundation of China (\#81272472).

\section{References}

1. Sun M, Jiang R, Li JD, et al: MED19 promotes proliferation and tumorigenesis of lung cancer. Mol Cell Biochem 355: 27-33, 2011.

2. Wang CG, Wang RY, Sun M, Li JD, Gao N and Zhang XY: In vivo antitumor effect of siRNA against STAT3 on transplanted Lewis lung cancer in mice. Chem Res Chin Univ 24: 322-329, 2008.

3. Luo SL, Sun M, Jiamg R, Wang G and Zhang XY: Establishment of primary mouse lung adenocarcinoma cell culture. Oncol Lett 2: 629-632, 2011.

4. Eymin B and Gazzeri S: Role of cell cycle regulators in lung carcinogenesis. Cell Adh Migr 4: 114-123, 2010.
5. Shintani S, Mihara M, Terakado N, et al: Reduction of p12DOC-1 expression is a negative prognostic indicator in patients with surgically resected oral squamous cell carcinoma. Clin Cancer Res 7: 2776-2782, 2001

6. Shintani S, Ohyama H, Zhang X, et al: p12(DOC-1) is a novel cyclin-dependent kinase 2-associated protein. Mol Cell Biol 20: 6300-6307, 2000.

7. Todd R, McBride J, Tsuji T, et al: Deleted in oral cancer-1 (doc-1), a novel oral tumor suppressor gene. FASEB J 9: 1362-1370, 1995.

8. Daigo Y, Suzuki K, Maruyama O, et al: Isolation, mapping and mutation analysis of a human cDNA homologous to the doc-1 gene of the Chinese hamster, a candidate tumor suppressor for oral cancer. Genes Chromosomes Cancer 20: 204-207, 1997.

9. Peng H, Shintani S, Kim Y and Wong DT: Loss of p12CDK2AP1 expression in human oral squamous cell carcinoma with disrupted transforming growth factor-beta-Smad signaling pathway. Neoplasia 8: 1028-1036, 2006.

10. Hiyoshi Y, Watanabe M, Hirashima K, et al: p12CDK2-AP1 is associated with tumor progression and a poor prognosis in esophageal squamous cell carcinoma. Oncol Rep 22: 35-39, 2009.

11. Zolochevska $\mathrm{O}$ and Figueiredo ML: Cell cycle regulator cdk2ap1 inhibits prostate cancer cell growth and modifies androgenresponsive pathway function. Prostate 69: 1586-1597, 2009.

12. Zolochevska $\mathrm{O}$ and Figueiredo ML: Expression of cell cycle regulator cdk2ap1 suppresses tumor cell phenotype by non-cellautonomous mechanisms. Oral Oncol 45: e106-112, 2009.

13. Zolochevska $\mathrm{O}$ and Figueiredo ML: Cell-cycle regulators cdk2ap1 and bicalutamide suppress malignant biological interactions between prostate cancer and bone cells. Prostate 71: 353-367, 2011.

14. Choi MG, Sohn TS, Park SB, et al: Decreased expression of p12 is associated with more advanced tumor invasion in human gastric cancer tissues. Eur Surg Res 42: 223-229, 2009.

15. Yuan Z, Gaba AG, Kent TS, Bennett A, Miller A and Weber TK: Modulation of CDK2-AP1 [p12(DOC-1)] expression in human colorectal cancer. Oncogene 24: 3657-3668, 2005.

16. Figueiredo ML, Kim Y, St John MA and Wong DT: p12CDK2AP1 gene therapy strategy inhibits tumor growth in an in vivo mouse model of head and neck cancer. Clin Cancer Res 11: 3939-3948, 2005

17. Cwikla SJ, Tsuji T, McBride J, Wong DT and Todd R: doc-1-mediated apoptosis in malignant hamster oral keratinocytes. J Oral Maxillofac Surg 58: 406-414, 2000.

18. Galetta D, Pisconti S, Cinieri S, et al: Induction pemetrexed and cisplatin followed by maintenance pemetrexed versus carboplatin plus paclitaxel plus bevacizumab followed by maintenance bevacizumab: a quality of life-oriented randomized phase III study in patients with advanced non-squamous non-small-cell lung cancer (ERACLE). Clin Lung Cancer 12: 402-406, 2011.

19. Zolochevska $O$ and Figueiredo ML: Novel tumor growth inhibition mechanism by cell cycle regulator cdk2ap1 involves antiangiogenesis modulation. Microvasc Res 80: 324-331, 2010.

20. Wong DT, Kim JJ, Khalid O, Sun HH and Kim Y: Double edge: CDK2AP1 in cell-cycle regulation and epigenetic regulation. J Dent Res 91: 235-241 2012.

21. Zheng J, Xue H, Wang T, et al: miR-21 downregulates the tumor suppressor P12 CDK2AP1 and stimulates cell proliferation and invasion. J Cell Biochem 112: 872-880, 2011.

22. Kim Y, McBride J, Kimlin L, Pae EK, Deshpande A and Wong DT: Targeted inactivation of p12, CDK2 associating protein 1, leads to early embryonic lethality. PLoS One 4: e4518, 2009.

23. Wadler S: Perspectives for cancer therapies with $\mathrm{cdk} 2$ inhibitors. Drug Resist Updat 4: 347-367, 2001.

24. Yang TY, Chang GC, Chen KC, et al: Sustained activation of ERK and Cdk2/cyclin-A signaling pathway by pemetrexed leading to $\mathrm{S}$-phase arrest and apoptosis in human non-small cell lung cancer A549 cells. Eur J Pharmacol 663: 17-26, 2011.

25. Kim Y, McBride J, Zhang R, Zhou X and Wong DT: p12(CDK2AP1) mediates DNA damage responses induced by cisplatin. Oncogene 24: 407-418, 2005. 